\title{
JURISPRUDENCIA AMBIENTAL EN ASTURIAS (PRIMER SEMESTRE 2020)
}

\author{
ALEJANDRA BOTO ÁLVAREZ \\ Profesora Titular de Derecho Administrativo \\ Universidad de Oviedo
}


Sumario: 1. La litigiosidad de los aprovechamientos en montes de utilidad pública.- 2. Telecomunicaciones y servidumbre de protección.- 3. Obras, actividad, apertura: ¿las apariencias engañan?

Para esta crónica se han elegido tres grupos de sentencias emanadas en los últimos meses de la sala de lo contencioso-administrativo del Tribunal Superior de Justicia de Asturias (TSJA) con relevancia ambiental. El primero está relacionado con los aprovechamientos en montes de utilidad pública, rodeados de una fuerte litigiosidad vecinal a que ya se ha hecho referencia en la crónica sobre derecho y políticas ambientales. La segunda de las sentencias tiene también cierta deriva demanial, esta vez en relación con instalaciones para telecomunicaciones en la zona de servidumbre de protección del dominio público marítimo-terrestre. La última de las sentencia analizadas se da en relación con las inmisiones acústicas de ciertas actividades, sobre las que existía un conflicto entre la apariencia y la autorización.

\section{LA LITIGIOSIDAD DE LOS APROVECHAMIENTOS EN MONTES DE UTILIDAD PÚBLICA}

En el apartado correspondiente a la crónica de derecho y políticas ambientales se ha dado cuenta de la aprobación del Plan Técnico Anual de Aprovechamientos para el año 2020, a ejecutar en los montes declarados de Utilidad Pública en el Principado de Asturias, que ha venido marcado por el resultado de distintos casos judiciales.

El primero procede de un enquistado litigio entre los vecinos de dos Concejos (Lena y Quirós) por los pastos en los montes del Aramo. La cuestión ha llegado varias veces ante el Tribunal Supremo, la última en 2013 al presentarse un recurso de casación frente a la sentencia del TSJA que había declarado la nulidad del Plan Técnico Anual de 20082. Es doctrina consolidada por esas sentencias, muy interesantes desde la perspectiva del valor de la costumbre en Derecho Administrativo, que los vecinos de Lena tienen derecho de pastoreo en

El trabajo ha sido elaborado en el marco del proyecto de investigación de referencia IDI/2018/000219 correspondiente a una ayuda a organismos públicos de investigación para apoyar las actividades de grupos de investigación en el Principado de Asturias concedida por el Gobierno de este al grupo SPAG (Servicios Públicos, Administraciones y Garantías) que coordina el profesor Tolivar Alas en la Universidad de Oviedo.

${ }_{2}$ STS 986/2013, de 12 de marzo. 
ciertos montes de utilidad pública del concejo de Quirós, pero siguen existiendo conflictos sobre la superficie de las majadas de esos pastoreos y la carga del aprovechamiento de pastos sobre las mismas.

Desde la última sentencia del Tribunal Supremo y hasta fechas recientes, las cargas propuestas por el Servicio de Montes permitieron lograr una cierta situación de equilibrio aceptada por ambas partes, cesando el continuado recurrir ante la justicia de los Planes Anuales sucedido entre 2002 y 2008, y acallando en cierta medida las múltiples desavenencias entre ambos Concejos. No obstante, el Plan de 2018 fue de nuevo impugnado, dando lugar a la sentencia del TSJA 705/2019, de 14 de octubre, que resuelve los asuntos acumulados del recurso 221/18, interpuesto por el Ayuntamiento de Quirós, y 252/18, interpuesto por el Ayuntamiento de Lena. Las respectivas pretensiones eran de anulaciones parciales de algunas disposiciones del Plan. El Tribunal falla la validez de los criterios establecidos para el reparto de la carga ganadera mancomunada entre los ganaderos de ambos Concejos, que se consideran ajustados a derecho, siendo esos criterios los que, con posterioridad, se han trasladado al Plan de 2019 y al Plan de 2020. Resta por ver si esta vez las impugnaciones van a parar aquí.

El Plan de 2020 está también marcado por otras dos sentencias. Así, la propuesta inicial del Plan de 2020 tuvo que corregirse acogiendo una alegación formulada en tiempo y forma que traía a colación la sentencia del TSJA 980/2019, de 27 de diciembre, dictada en procedimiento ordinario 451/2019. El recurso había sido interpuesto por la Unión de Campesinos Asturianos (UCAUPA), contra una Resolución de acotamiento al pastoreo de la Consejería, siendo codemandado el Ayuntamiento de Aller. El pleito versa sobre un acotamiento posterior a un incendio, materia en la que se ha dado una reciente modificación legislativa, tratada en la crónica de derecho y políticas ambientales en Asturias de 2017 (vol. VIII, núm. 1). Tal modificación está en el fondo del litigio y así el TSJ la analiza, para precisar que el sentido de la modificación legal previa de la normativa estatal de montes es la eliminación de la imposición automática de la medida impugnada en caso de incendio, puesto que la experiencia ha demostrado que la obligación de acotamiento no es necesaria en todos los casos para procurar la recuperación de la cubierta vegetal quemada, ni para lograr la 
restitución de las especies afectadas a la situación anterior al mismo, sino que se deja al prudente arbitrio de los responsables de las Comunidades Autónomas la determinación de la toma de esa radical medida en los casos y por el tiempo que sea imprescindible para la recuperación de las especies afectadas. Así, desde 2017 en Asturias esta medida se sujeta a determinadas condiciones relacionadas con la calificación del incendio y de la superficie afectada, y cuando afecta arbolado resulta técnicamente incuestionable la incompatibilidad de su regeneración con la realización de determinadas actividades, entre ellas el pastoreo, por lo que en estos casos el acotamiento tiene cobertura legal y material.

Sentado esto, la dificultad surge, como ponen de manifiesto las dispares opiniones de las partes litigantes con base en un informe pericial y en las declaraciones de sus servicios técnicos, en la aplicación práctica de estos requisitos legales, dado que para concretar la definición de monte arbolado se acude a parámetros establecidos en inventarios y boletines oficiales sobre lo que es un bosque o un monte. Dependiendo de esos factores y de los datos concretos del incendio, la determinación y cálculo de las superficies arboladas afectadas por el incendio, y con ello la limitación periódica de las zonas acotadas, pueden dar lugar a resultados muy divergentes, por más que las respectivas partes los consideren reflejo de la realidad. Incluso aceptado que el acotamiento sea necesario, pueden surgir también desavenencias sobre su extensión espacial y temporal.

En el caso, valorados los medios de prueba practicados resulta claro, en primer lugar, que ha tenido lugar un incendio, que comprende entre la superficie incendiada en monte bajo y monte alto una masa arbórea con diferente grado de desarrollo, pero también otra superficie de matorral y prado arbustivo. Para el Tribunal no queda clara la delimitación entre una superficie y otra. Como la medida de acotamiento para la actividad ganadera que se recurre se acordó por la Administración para la regeneración intensiva de árboles, y esa afección no ha quedado clara, el Tribunal estima el recurso sin condena en costas.

En consecuencia, el acotamiento tiene que desaparecer del Plan Técnico de Aprovechamientos y las superficies que figuraban afectadas por dicho acotamiento declarado nulo, han de ser también incluidas en la superficie 
admisible a pastos a efectos de la concesión de licencias de pastos y ayudas de la PAC.

Finalmente, en el proceso de elaboración del Plan Técnico de Aprovechamientos para 2020 fue en cambio desestimada otra alegación fundada en la sentencia del TSJA 813/2019, de 11 de noviembre, dictada en procedimiento ordinario 419/2018. El recurso se había presentado por el Ayuntamiento de Teverga frente al deslinde de un monte de utilidad pública sito en su concejo, por el que la Consejería había trazado la línea divisoria de uso del monte que correspondía a dos núcleos, y está relacionada con una sentencia anterior también del TSJA (la 119/2019, de 18 de febrero, dictada en procedimiento ordinario 79/2018) en que una Parroquia rural del Concejo había impugnado ese mismo deslinde, siendo su pretensión estimada. De nuevo pueden verse los evidentes conflictos enquistados entre vecinos, esta vez dentro del mismo municipio. La sentencia de febrero había determinado la necesidad de repetir el deslinde bajo determinados criterios que la misma recoge, mientras que la sentencia de noviembre considera la cuestión planteada, con el mismo fondo que lo ya ventilado en el primer recurso, como cosa juzgada. Es decir, persiste la necesidad de repetir el procedimiento técnico-legal del deslinde administrativo de las partes del monte que podrían corresponder a cada entidad.

Ello había llevado a la Parroquia rural a realizar alegaciones al borrador del Plan Técnico Anual de Aprovechamientos para 2020 para que se le atribuyera en él la superficie exacta que le correspondiera. Pero la alegación fue desestimada, por entenderse que el monte se encuentra en deslinde parcial y en tanto no se resuelva el mismo, la carga ganadera se repartirá de forma mancomunada entre la Parroquia rural y el Ayuntamiento.

\section{TELECOMUNICACIONES Y SERVIDUMBRE DE PROTECCIÓN}

En sentencia 942/2019, de 13 de diciembre, el TSJA resuelve el recurso número 70/2019, presentado contra una Resolución de 3 de diciembre de 2018 de la Dirección General de Infraestructuras, Ordenación del Territorio y Medio Ambiente del Principado de Asturias que denegaba para una estación base de telefonía móvil a colocar dentro de la zona de servidumbre del dominio público marítimo-terrestre. 
La parte demandante sostenía que se trataba de una instalación compatible con la normativa y autorizable, al estar vinculada la instalación al dominio público marítimo y uso necesario para su servicio. Existe, entiende, necesidad o conveniencia acreditada, siendo las playas a las que serviría la instalación zona de descanso y esparcimiento en las que se concentra gran cantidad de personas durante una determinada época del año, y pareciendo lógico que cuenten con determinados servicios, entre los cuales el uso de los dispositivos móviles. Censuraba además la falta, en la tramitación administrativa de la autorización, de informe del Ministerio de Economía y Empresa. Al examinar este punto, el TSJA encuentra que en efecto el informe era preceptivo de acuerdo el artículo 35.5 de la Ley 9/2014, de 9 de mayo, General de Comunicaciones.

Indiscutido el carácter no sólo preceptivo, sino esencial del citado informe con finalidad de contar con una información cualificada sobre la necesidad de la infraestructura que se cuestiona para el despliegue de las redes públicas de comunicaciones electrónicas, se aprecia que en su momento la Administración demandada solicitó el informe y el Ministerio interesó la remisión de determinada documentación. Una vez enviada y entregada esa documentación, transcurrió el plazo de un mes para la emisión del informe sin hacerlo, ante lo que la Administración autonómica continuó con la tramitación, al entender emitido con carácter favorable. La actuación fue correcta y no da lugar a nulidad (Fundamento tercero).

Para la Administración, la instalación podría perfectamente situarse fuera de la zona de servidumbre, como otras que ya existen en los alrededores. Obviamente, recuerda, las estaciones de telefonía móvil no están directa ni indirectamente vinculadas al medio marino, ni es necesario que se implanten sobre el demanio marítimo-terrestre y su servidumbre de protección. Y finalmente, se aduce que la instalación de una estación de telefonía móvil no

\footnotetext{
3 Que trae causa en la competencia exclusiva estatal en materia de telecomunicaciones del artículo 149.1.21. a de la Constitución y en las competencias de carácter transversal de los artículos 149.1.1. ${ }^{a}$ y 149.1.13. ${ }^{a}$ del Texto constitucional, con las que esta Ley persigue, como uno de sus principales objetivos, el de recuperar la unidad de mercado en el sector de las telecomunicaciones, estableciendo procedimientos de coordinación y resolución de conflictos entre la legislación sectorial estatal y la legislación de las Administraciones competentes dictada en el ejercicio de sus competencias que pueda afectar al despliegue de redes y a la prestación de servicios
} 
presta un servicio necesario o conveniente para el uso del dominio público marítimo-terrestre, sino para el uso del teléfono móvil, cuestión sumamente distinta. Según su postura, la estación es innecesaria, en la pretendida ubicación, para garantizar la cobertura.

A este respecto, el TSJA encuentra que las razones dadas por ambas partes resultan "objetivamente razonables", atendiendo a los objetivos y fines de la protección del dominio público que impone una limitación de sus usos y a la calidad en la prestación de un servicio de interés general y público, en relación a determinados servicios, de ahí su especial importancia para la población. Se inclina ante ello y en este caso por acoger el criterio de la parte demandante, que cuenta con la justificación y explicación concreta de que con la instalación se dota de buena calidad y cobertura en las playas, así como a los pueblos colindantes dada la ubicación estratégica de la antena, que excede a las meras razones de oportunidad que alega la parte demandada, para lo cual se basa en las instalaciones que tienen otras sociedades operadoras de telecomunicaciones fuera de la zona sin afectar a la calidad del servicio, ya que alejarse de los objetivos principales de cobertura (playas), supondría una degradación de esa calidad dada la filosofía de las redes móviles, mientras que la protección de costas no puede impedir esta clase de instalaciones como las que nos ocupan, pues tal protección ha de resultar compatible con la existencia de instalaciones que resulten convenientes en las costas (Fundamento cuarto).

\section{OBRAS, ACTIVIDAD, APERTURA: ¿LAS APARIENCIAS ENGAÑAN?}

El último de los fallos a analizar en estas páginas, de 31 de enero de 2020, resuelve el recurso de apelación 347/2019, presentado contra sentencia de 11 de octubre de 2019 del Juzgado contencioso-administrativo número 6 de Oviedo. La sentencia de instancia había desestimado a su vez el recurso en procedimiento ordinario número $2 / 19$ instado por la parte recurrente (una comunidad de propietarios) contra dos resoluciones municipales que otorgaban a un tercero sendas licencias para apertura de local destinado a la actividad de taller de mecánica rápida y de venta menor de neumáticos y montaje en la misma ubicación. 
El argumento del recurso se fundaba en que el Ayuntamiento había denominado únicamente "licencias de obra" a sus resoluciones anteriores en el expediente, lo que generó en los administrados confianza en que lo que se está autorizando en ese momento eran sólo obras, no la instalación de la actividad. Adaptaron su conducta a la apariencia creada por la propia Administración y no reaccionaron en plazo durante la tramitación de la autorización como lo habrían hecho de haber sabido que estaban ante una licencia de actividad, que esperaban que se tramitara después. Sin embargo, y sin ese procedimiento intermedio, el Ayuntamiento concedió directamente la licencia de apertura, frente a lo que acudieron ante el juzgado en primera instancia alegando fraude de ley y omisión del procedimiento establecido, argumentos que no fueron acogidos. En apelación argumentan que la sentencia de instancia no ha aplicado adecuadamente el principio de protección de la confianza legítima y, por tanto, debe ser revocada en este punto. También se denuncia que la omisión del trámite de información pública vicia no solo el otorgamiento de la licencia de actividad, sino inevitablemente también la concesión de la licencia de apertura. En cuanto al fondo, consideran que se incurre en error en la apreciación de la prueba, con infracción del artículo 22 del Reglamento de Servicios de las Corporaciones Locales y del artículo 3.4 del Anexo IV del Real Decreto 1367/2007, de 19 de octubre, por falta de representatividad de los dos informes de medición acústica aportados por el promotor para obtener la licencia de apertura, al no ajustarse ninguno de tales informes a las exigencias del referido Anexo IV, pues la medición no se realizó con un mínimo de tres posiciones, ni tampoco en periodo diurno, sino cuando era ya de noche, entre las 19:41 y las 20:18 horas del 8 de febrero de 2018, que no se corresponde con el de mayor influencia de la actividad, según los proyectos que han obtenido la licencia.

Al recurso de apelación se opone la Corporación Municipal demandada, negando que se haya hecho una errónea interpretación y aplicación de la norma jurídica aplicable y defendiendo que por el Ayuntamiento se han respetado los principios de buena fe y protección de la confianza legítima en su actuar a lo largo del expediente administrativo con cumplimiento de todos los requisitos técnicos, urbanísticos y ambientales exigidos. En su opinión también se debe desestimar la pretensión apelante en lo que se refiere a las molestias por ruido, 
ya que los informes de medición se ajustan a lo exigido en el Real Decreto 1367/2007 y, al no existir informe técnico en contrario que lo desvirtúe, procede entender que se cumplen todas las medidas correctoras impuestas; por lo que se interesa que se dicte sentencia desestimatoria del recurso de apelación interpuesto, confirmando la sentencia de instancia.

EI TSJA entiende que la sentencia apelada no ha incurrido en la errónea interpretación de la normativa sectorial que aplica que se denuncia, pues examinando el iter procedimental seguido distingue claramente las dos fases que comprende el procedimiento de autorización de una actividad clasificada como la litigiosa: por un lado la de actividad, englobada en la de obras, y por otro la de apertura, que constituye el objeto de recurso, razón por la que la instancia, con acertado criterio, no acoge la denuncia de haberse omitido la información pública y notificación a colindantes, pues son motivos de impugnación que debieron haberse hecho valer respecto a la licencia de actividad, que sería cuestión ajena a este recurso, circunscrito a las licencias de apertura.

Ello debe entenderse así, sin merma de los principios de buena fe y protección de la confianza legítima que invoca la apelante, pues de un lado no es posible invocar con éxito una indefensión ajena, esto es, la indefensión a que se refiere el artículo 48.2 de la Ley 39/2015, de 1 de octubre, del Procedimiento Administrativo Común de las Administraciones Públicas, que es el que contempla los defectos de forma, indefensión que es la de los "interesados" y no la que eventualmente hayan sufrido terceros, dato que se resalta porque no se aprecia que a la comunidad apelante se le haya menoscabado el derecho de defensa, cuando paladinamente reconoce que tuvo conocimiento de las resoluciones de otorgamiento de las licencias de obras para la adecuación de local para taller de mecánica rápida y de venta y reparación de neumáticos (...), absteniéndose de impugnarlas y aguardando a que se otorgare la futura licencia de actividad; y de otra parte, porque las licencias de obras concedidas en su día comprenden ineludiblemente la autorización para desarrollar las actividades solicitadas, tal como se desprende de sus respectivos contenidos, que aluden a su calificación de actividades clasificadas y que, por ello, fueron previamente informadas por el Servicio de Autorizaciones 
Ambientales de la Consejería correspondiente, imponiendo las condiciones a cuyo cumplimiento quedaban condicionadas las actividades, que no las obras, a desarrollar, lo que se explica desde una lógica jurídica elemental, pues carecería de sentido conceder el permiso de obras sin el otorgamiento de la licencia de actividad, con lo que se configura una interdependencia y un orden de prelación entre ambas licencias que están proyectados sobre el principio de una hipotética responsabilidad por el posible funcionamiento anormal de la Administración en la inobservancia de la precedencia temporal señalada, precedencia que se establece principalmente en interés del particular afectado, a fin de evitar los gastos de la ejecución de una obra de la que no pudiera obtenerse la utilidad esperada por no ser susceptible de destinarse a la actividad para la que fue proyectada (SSTS 18 junio 1990, 2 octubre 1995 y 17 mayo 1999). En consecuencia, la falta de una indefensión efectiva determina asimismo que no tenga la virtualidad pretendida el dato de haberse emitido informes sobre las actividades clasificadas pretendidas antes de haberse formulado alegaciones que, de hecho, no se presentaron, pudiendo la parte haberlo efectuado 4.

Idéntica suerte desestimatoria merece el motivo del recurso referido al error en la apreciación de la prueba en que a juicio de la apelante incurre la sentencia apelada, por la doctrina jurisprudencial que impide la revisión en segunda instancia de la valoración del acervo probatorio efectuada por el Juez a quo excepto en caso de que la misma sea absurda o claramente errónea. EI TSJA no entiende que tal cosa concurra en el presente supuesto. De hecho, la Sala comparte el punto de vista de la Juzgadora de instancia en orden a que la prueba existente acredita que se cumplen todas las medidas correctoras que se impusieron en la licencia de actividad, incluidas las medidas de protección para evitar las molestias por ruido; $y$, toda vez que no se ha practicado a instancia de la recurrente informe técnico alguno que desvirtúe los informes de medición aportados, han de prevalecer estos aunque no sea más que por la falta de informe contradictorio (Fundamento cuarto). 
En definitiva, no se acoge ninguno de los motivos de la apelación, que en general resultan una reiteración de los argumentos que ya fueron rechazados en la instancia. Por ello se desestima el recurso con una condena en costas con el límite de 1.000 euros por todos los conceptos, habida cuenta la complejidad del asunto y el esfuerzo dialectico que ha tenido que ser empleado por la Corporación municipal para articular su defensa (Fundamento sexto)

Todo ello sin desconocer que la licencia otorgada genera una relación permanente con la Administración que siempre podrá acordar lo preciso para que la actividad se ajuste a las exigencias de interés público que condicionan implícitamente este tipo de licencias. 